Physica 135A(1986) 519-532

North-Holland, Amsterdam

\title{
ROTATIONAL ORDERING AND SYMMETRY BREAKING IN THE TRIANGULAR ANTIFERROMAGNETIC HEISENBERG LATTICE
}

\author{
W.J. CASPERS
}

Center for Theoretical Physics, Twente University of Technology, Enschede, The Netherlands

and

\section{G.I. TIELEN}

Zelhem, The Netherlands

Received 18 September 1985

Finite system calculations suggest a rotational ordering in the ground state of the triangular antiferromagnetic Heisenberg lattice. This rotational ordering may result in a rotational symmetry breaking for quantum-spin systems, which does not show a net sublattice magnetization.

\section{Introduction}

The classical, intuitive picture of magnetic ordering is intimately connected with different forms of symmetry breaking (SB). In the case of ferromagnetism, for which neighbouring spins have the tendency to be oriented in a parallel direction, there is still an arbitrariness in the overall spin direction, at least for an isotropic interaction between the spins. This arbitrariness results in breaking of the spherical symmetry for which all directions of the total magnetization (or spin) are equivalent. In antiferromagnetic systems the existence of two sublattices with antiparallel orientation results in a form of SB connected with the translational symmetry of the interaction. It is still an open question whether or not this intuitive picture of antiferromagnetism (AF) is confirmed by exact calculations for well-defined models.

The only exact results known so far are for one-dimensional systems of the $X Y$-type, for which the well-known Jordan-Wigner transformation gives the possibility of evaluating the spin correlation functions ${ }^{1-3}$ ). It turns out that only in the anisotropic case there is a long-range order. Numerical calculations for 
Heisenberg chains suggest an analogous situation: only interactions of cylindrical (or lower) symmetry, with a preferred direction, show long-range order in the ground state ${ }^{4}$ ).

Exact results for two-dimensional systems of arbitrary size are not known so far, apart from the completely solvable Ising model, but a theorem of Mermin and Wagner ${ }^{5}$ ) predicts the absence of long-range order in any system with an isotropic Heisenberg interaction in the case of one and two dimensions, at least for finite temperatures.

Apart from SB that results in a net sublattice magnetization, certain onedimensional models have a so-called singlet-pair state (SPS) as their ground state $^{6-9}$ ). The spins in this state are ordered in neighbouring spin pairs, which are all in the singlet state. This can be realized in two different ways resulting in a SB for the discrete group of lattice translations, without a net sublattice magnetization. Whereas for the continuous SB that results in a sublattice magnetization, there exists always a soft mode in the spectrum of elementary excitations, according to Goldstone's theorem ${ }^{10}$ ), the "discrete" SB may give rise to a lower bound in this spectrum. The existence of such a lower bound seems to be confirmed by actual calculations for the Majumdar-Ghosh chain with isotropic interactions between nearest and next-nearest neighbour spins, with a strength in the ratio $2: 1^{11,12}$ ).

Characteristic for the Majumdar-Ghosh chain is the existence of frustration in the corresponding Ising model (in which only the coupling in the $z$-direction is taken into consideration). This frustration results in a highly degenerate Ising ground state. Then one may put the general question: is there a relation between frustration and the existence of a Heisenberg groundstate that shows an ordering that is of a different nature than the one of the well-known Néel type? This question led us to the study of the triangular antiferromagnetic Heisenberg lattice, for which the corresponding Ising problem has been studied in the literature ${ }^{13,14}$ ).

Calculations for the ground state of small groups of neighbouring spins $\frac{1}{2}$ on the triangular lattice strongly suggest the phenomenon of rotational correlation (RC). This concept is introduced in section 2 , in which simple calculations are performed for a triangle and a diamond. In section 3 we show that this concept is also useful for larger systems, in particular with 12 spins. For the last system exact calculations were performed already by Marland and Betts ${ }^{15}$ ).

The concept of RC suggests the possibility of rotational symmetry breaking (RSB) for large systems. Section 4 is devoted to this possibility, which may result in long-range order (LRO) without a net sublattice magnetization. We do not answer the question whether or not this RSB is stable at finite temperatures, or may be destroyed by an analogous phenomenon as described by Mermin and Wagner l.c. 
In the examples we have studied it is easy to show that the exact ground state is a linear combination of two states with different RSB. We expect that states of different RSB are asymptotically orthogonal for large systems, so the phenomenon of RSB for the ground state appears in a natural way.

\section{Examples of ground states with 3 and 4 spins}

In the discussion of our ordering principle it is necessary to make a subdivision of the lattice into three sublattices, denoted by the letters $a, b$ and $c$ (fig. 1). The spins in the different examples, being distinguishable objects are numbered 1,2, $3,4, \ldots$ In the illustrations of these examples the spins as well as the sublattices are indicated. The interaction will be given by the general Hamiltonian

$$
H=4 \sum_{\langle i, j\rangle}^{\prime} S_{i} \cdot S_{j}
$$

in which expression the $\boldsymbol{S}_{i}\left(\boldsymbol{S}_{j}\right)$ denote Pauli spins for the lattice sites $i(j)$ and $\Sigma_{\langle i, j\rangle}^{\prime}$ indicates a sum over all neighbour pairs. It seems natural to study only clusters of neighbour spins confined within a convex polygon.

\subsection{The equilateral triangle}

This simple system is illustrated by fig. 2 and its dynamics is given by the Hamiltonian

$$
H=4\left(S_{1} \cdot S_{2}+S_{2} \cdot S_{3}+S_{3} \cdot S_{1}\right)=2\left(S^{2}-3 \cdot \frac{3}{4}\right), \quad S=S_{1}+S_{2}+S_{3} .
$$

This Hamiltonian is easily diagonalized and gives a fourfold degenerate ground

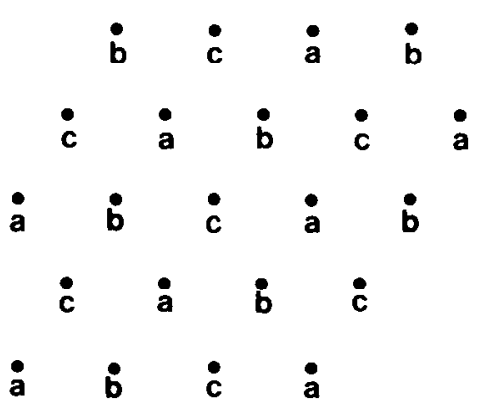

Fig. 1. Sublattices in the triangular lattice. 


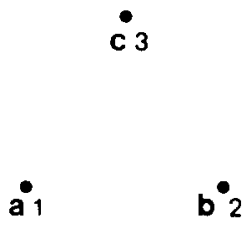

Fig. 2. The equilateral triangle.

state:

$$
\begin{aligned}
& \left|\lambda,-\frac{1}{2}\right\rangle=\frac{1}{\sqrt{3}}\left[|+--\rangle+\lambda|-+-\rangle+\lambda^{-1}|--+\rangle\right], \\
& \left|\lambda,+\frac{1}{2}\right\rangle=\frac{1}{\sqrt{3}}\left[|-++\rangle+\lambda|++-\rangle+\lambda^{-1}|+-+\rangle\right], \\
& \left|\lambda^{-1},-\frac{1}{2}\right\rangle=\frac{1}{\sqrt{3}}\left[|+--\rangle+\lambda^{-1}|-+-\rangle+\lambda|--+\rangle\right], \\
& \left|\lambda^{-1},+\frac{1}{2}\right\rangle=\frac{1}{\sqrt{3}}\left[|-++\rangle+\lambda^{-1}|++-\rangle+\lambda|+-+\rangle\right], \\
& \lambda=\mathrm{e}^{2 \pi i / 3}, \quad \lambda^{-1}=\mathrm{e}^{-2 \pi \mathrm{i} / 3}, \quad 1+\lambda+\lambda^{-1}=0 .
\end{aligned}
$$

In (3) the kets like $|+--\rangle$ denote a state for which spin 1 is in the eigenstate of $S_{1 z}$ with eigenvalue $\frac{1}{2}(\hbar=1)$, for spin $2(\operatorname{spin} 3)$ the eigenvalue of $S_{2 z}\left(S_{3 z}\right)$ equals $-\frac{1}{2}$ etc. All four linear combinations in (3) have total spin $S=\frac{1}{2}$, but its $z$-component $S_{z}=S_{1 z}+S_{2 z}+S_{3 z}$ has the eigenvalues $M=-\frac{1}{2},+\frac{1}{2},-\frac{1}{2},+\frac{1}{2}$ respectively. The four states belong to two different representations of the rotation group for the threefold axis. The eigenvalue for the symmetry operation $(1 \rightarrow 2,2 \rightarrow 3,3 \rightarrow 1)$ equals $\lambda^{-1}, \lambda, \lambda, \lambda^{-1}$ respectively. The index $\lambda\left(\lambda^{-1}\right)$ in the left-hand sides, however, has a different meaning, more suitable for a definition of RSB.

The meaning of this index will be illustrated by the state $\left|\lambda,-\frac{1}{2}\right\rangle$. One considers the interchange of a + spin on the site $a$ and a - spin on the site $b$, or + on $b$ and - on $c$, or + on $c$ and - on $a$ : if these interchanges are followed by multiplication with a factor $\lambda$ the original state is unchanged.

The same is true for $\left|\lambda,+\frac{1}{2}\right\rangle$, whereas, for the same operation the states $\left|\lambda^{-1},+\frac{1}{2}\right\rangle$ should be multiplied by $\lambda^{-1}$ to be left unchanged. It should be kept in mind that $\left|\lambda,-\frac{1}{2}\right\rangle$ and $-\left|\lambda^{-1},+\frac{1}{2}\right\rangle$ are transformed into each other by the step operators

$$
S^{+}=S_{1}^{+}+S_{2}^{+}+S_{3}^{+} \quad\left(S_{1}^{+}=S_{1}^{x}+\mathrm{i} S_{1}^{y}\right)
$$

and

$$
S^{-}=S_{1}^{-}+S_{2}^{-}+S_{3}^{-} \quad\left(S_{1}^{-}=\left(S_{1}^{+}\right)^{+}\right),
$$


and the same is true for $\left|\lambda^{-1},-\frac{1}{2}\right\rangle$ and $-\left|\lambda,+\frac{1}{2}\right\rangle$, as may be checked easily. The eigenvalue of $H$ for all 4 states equals: $2\left(\frac{3}{4}-\frac{9}{4}\right)=-3$. The ground state is in this case fourfold; it shows apart from Kramers' degeneracy an additional degeneracy corresponding with RSB. Time-reversal symmetry transforms the states $\left|\lambda,-\frac{1}{2}\right\rangle$ and $\left|\lambda,+\frac{1}{2}\right\rangle$ into one another and the same is true for the other two states. The two doublets correspond with different RSB, and only in this simple example the RSB leads to exact eigenstates of $H$, which are also orthogonal. The index $\lambda$ is a label for the rotational character, which for this simple example may be connected with an irreducible representation of an (Abelian) group, in contradistinction with the more complicated cases to be discussed hereafter.

\subsection{The diamond}

Here the situation is much more interesting than for the simple triangle. Now there is only one spin on an $a$ or $b$ site, whereas there are two spins on $c$ sites (fig. $3)$. First we solve exactly the relatively simple eigenvalue problem for the Hamiltonian

$$
\begin{aligned}
H & =4\left(S_{1} \cdot S_{2}+S_{2} \cdot S_{3}+S_{3} \cdot S_{4}+S_{4} \cdot S_{1}+S_{2} \cdot S_{4}\right) \\
& =4\left[\left(S_{1}+S_{3}\right) \cdot\left(S_{2}+S_{4}\right)+S_{2} \cdot S_{4}\right] \\
& =4 S_{\mathrm{I}} \cdot S_{\mathrm{II}}+2 S_{\mathrm{II}}^{2}-3=2\left(S^{2}-S_{\mathrm{I}}^{2}\right)-3 \\
S_{\mathrm{I}} & =S_{1}+S_{3}, \quad S_{\mathrm{II}}=S_{2}+S_{4} .
\end{aligned}
$$

Because of the commutation relations: $\left[S^{2}, S_{\mathrm{I}}^{2}\right]=\left[S^{2}, S_{\mathrm{II}}^{2}\right]=0$, the system has three good quantum numbers corresponding with the eigenvalues of $S^{2}, S_{1}^{2}$ and $S_{\mathrm{II}}^{2}$ respectively. These quantum numbers will be denoted by $S, S_{\mathrm{I}}$ and $S_{\mathrm{II}}$ and the corresponding eigenvalues are $S(S+1)$, etc. $(\hbar=1)$. In table I we give all the

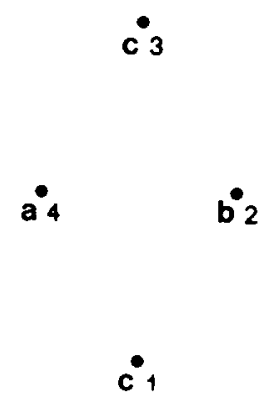

Fig. 3. The diamond 
Table I

Quantum numbers and eigenvalues of $H$.

\begin{tabular}{lllll}
\hline$S$ & $S_{\text {I }}$ & $S_{\text {II }}$ & \multicolumn{1}{c}{$E$} & Degeneracy \\
\hline 0 & 0 & 0 & -3 & 1 \\
1 & 0 & 1 & 1 & 3 \\
1 & 1 & 0 & -3 & 3 \\
0 & 1 & 1 & -7 & 1 \\
1 & 1 & 1 & -3 & 3 \\
2 & 1 & 1 & 5 & 5 \\
\hline
\end{tabular}

Table II

States of spin pairs.

\begin{tabular}{llc}
\hline & $S_{\mathrm{I}(\mathrm{II})}$ & $M_{\mathrm{I}(\mathrm{II})}$ \\
\hline$|0\rangle=\frac{1}{\sqrt{2}}[|+\rangle$ & $+\rangle]$ & 0 \\
$|11\rangle=|++\rangle$ & 1 & 1 \\
$|10\rangle=\frac{1}{\sqrt{2}}[|+-\rangle+|-+\rangle]$ & 1 & 0 \\
$|1-1\rangle=|--\rangle$ & 1 & -1 \\
\hline
\end{tabular}

eigenvalues $H(E)$, which have a degeneracy corresponding with the possible different eigenvalues of one of the components of $S$, e.g. $S_{2}$, which again commutes with $S^{2}, S_{1}^{2}$ and $S_{11}^{2}$.

The ground state, corresponding with the eigenvalue $E=-7$, is easily constructed with the help of the technique of coupling of angular momenta ${ }^{16}$ ). The four two-spin states corresponding with a total spin $\left(S_{\mathrm{I}}\right.$ or $\left.S_{\mathrm{II}}\right)$ that equals 0 or 1 , are given in table II. The + and - signs in the kets $|+,-\rangle$, etc. denote the eigenvalues $\pm \frac{1}{2}$ of $S_{1 z}$ and $S_{3 z}$ (or $S_{2 z}$ and $S_{4 z}$ ) and the quantum numbers $S_{\text {I(II) }}$ and $M_{\mathrm{I}(\mathrm{II})}$ pertain to the total spin and its $z$-component.

The state vector of the total system for its ground state has a (total) spin $S=0$, and $S_{\mathrm{I}}=S_{\mathrm{II}}=1$, so, according to the rules of coupling of angular momenta, it takes the form

$$
|\rangle=\sum_{M=-1}^{+1}\langle 1 M 1-M \mid 1100\rangle|1 M\rangle_{\mathrm{I}}|1-M\rangle_{\mathrm{II}},
$$

in which $\langle 1 M 1-M \mid 1100\rangle$ is a vector coupling (VC) or Clebsch-Gordan coefficient (cf. ref. 16, p. 37) and I and II refer to the spin pairs $(1,3)$ and $(2,4)$ respectively. The values of the particular $\mathrm{VC}$ coefficients used here may be found in ref. 16 formula (3.5.13), p. 40 :

$$
\langle 1 M 1-M \mid 1100\rangle=\frac{(-)^{1-M}}{\sqrt{3}},
$$

and the state | > may now be written out explicitly:

$$
\begin{aligned}
|\rangle= & \frac{1}{\sqrt{3}}\left[|+-+-\rangle+|-+-+\rangle-\frac{1}{2}(|++--\rangle+|+--+\rangle\right. \\
& +|-++-\rangle+|--++\rangle)],
\end{aligned}
$$

in which the positions in the kets in the right-hand side refer to spins 1,2,3 and 4 respectively (cf. fig. 3 ). 
One may gain insight into the phenomenon of RSB by considering (6) as a superposition of two states of different rotational character, or states with a broken rotational symmetry:

$$
\begin{aligned}
|\lambda\rangle= & x(\lambda)(|+-+-\rangle+|-+-+\rangle)+y(\lambda)[\lambda(|+--+\rangle \\
& \left.+|--++\rangle)+\lambda^{-1}(|++--\rangle+|-++-\rangle)\right], \\
\left|\lambda^{-1}\right\rangle= & x\left(\lambda^{-1}\right)(|+-+-\rangle+|-+-+\rangle)+y\left(\lambda^{-1}\right)\left[\lambda^{-1}(|+--+\rangle\right. \\
& +|--++\rangle)+\lambda(|++--\rangle+|-++-\rangle)] .
\end{aligned}
$$

In (7) $x(\lambda), y(\lambda), x\left(\lambda^{-1}\right)$ and $y\left(\lambda^{-1}\right)$ represent positive numbers. The rotational character of state $|\lambda\rangle$ is defined by the following rule: If in a ket a + spin is transferred from an $a$ to a $b$ position with the simultaneous transfer of a - spin in the opposite direction, this ket should be multiplied by a complex factor with argument $2 \pi / 3$, i.e. by $c_{0} \lambda$ with $c_{0}>0$. The same recipe should be used for interchange of $(+-)$ pairs on $(b, c)$ or $(c, a)$ positions. It should be clear that there is a factor $c_{0}^{\prime} \lambda^{-1}\left(c_{0}^{\prime}>0\right)$ for the inverse transpositions.

For the state $\left|\lambda^{-1}\right\rangle$ there is an analogous recipe with $\lambda$ replaced by $\lambda^{-1}$. The states in (3) have a rotational character given by the index $\lambda\left(\lambda^{-1}\right)$ in the symbols $\left|\lambda,-\frac{1}{2}\right\rangle$, etc.

One may introduce more positive constants in $|\lambda\rangle$ and $\left|\lambda^{-1}\right\rangle$ to define a still more general state of broken rotational symmetry, but this would not serve our purpose of mere illustration of the phenomenon of RSB.

In order to write $\rangle$ as a linear combination of states of the type $|\lambda\rangle,\left|\lambda^{-1}\right\rangle$ we have to take

$$
|\rangle=\frac{1}{\sqrt{2}}\left[|\lambda\rangle+\left|\lambda^{-1}\right\rangle\right], \quad x(\lambda)=y(\lambda)=x\left(\lambda^{-1}\right)=y\left(\lambda^{-1}\right)=\frac{1}{\sqrt{6}} .
$$

One easily verifies that also for this simple example: $\left\langle\lambda \mid \lambda^{-1}\right\rangle=0$. It should be stressed, however, that, in general, states of different rotational character need not be orthogonal. So far the expression "rotational character" has a rather loose meaning and only refers to the comlex phases of the coefficients of the Ising kets in a state vector like the ones given in (7). With a given "rotational character" there is not connected, in general, a good quantum number.

Now it is interesting to calculate the expectation value of the energy $H$ given in (4), for the states $|\lambda\rangle,\left|\lambda^{-1}\right\rangle$ as a function of $x$ and $y$, under the normalization condition 


$$
2 x^{2}(\lambda)+4 y^{2}(\lambda)=2 x^{2}\left(\lambda^{-1}\right)+4 y^{2}\left(\lambda^{-1}\right)=1,
$$

which reduces the number of independent variables to 1. An elementary calculation gives

$$
\langle\lambda|H| \lambda\rangle=-6 x^{2}(\lambda)-16 x(\lambda) y(\lambda)-8 y^{2}(\lambda),
$$

with an analogous expression for $\left\langle\lambda^{-1}|H| \lambda^{-1}\right\rangle$. The minimum value of $\langle\lambda|H| \lambda\rangle$, under the condition (9), is simply found to be

$$
\begin{aligned}
& -\frac{1}{2}(5+\sqrt{33})=-5.372, \\
& x(\lambda)=\left[\frac{33+\sqrt{33}}{132}\right]^{1 / 2}, y(\lambda)=\frac{1}{2}\left[\frac{33-\sqrt{33}}{66}\right]^{1 / 2},
\end{aligned}
$$

whereas its value for $x(\lambda)=y(\lambda)=1 / \sqrt{6}$ equals -5 . So we may distinguish between:

- the exact ground state

$$
|\rangle=\frac{1}{\sqrt{2}}\left[|\lambda\rangle+\left|\lambda^{-1}\right\rangle\right], \quad\left\langle\lambda \mid \lambda^{-1}\right\rangle=0, \quad E=-7,
$$

- optimalized states with given RSB, according to (11),

$$
\left\langle\lambda \mid \lambda^{-1}\right\rangle \neq 0, \quad E=-5.372,
$$

- orthogonal states with given RSB,

$$
\left\langle\lambda \mid \lambda^{-1}\right\rangle=0, \quad E=-5
$$

In section 4 we will come back to this concept of RSB in more detail. Here we may pay some more attention to the phenomenon of rotational correlation (RC), which is shown by the states (6) as well as (7). So far we have connected the rotational character of a state with the complex phases of the Ising kets. It is easier to introduce an operator, the expectation value of which is related with this rotational character. For a configuration like the one in fig. 2 we first define the operator

$$
\begin{aligned}
A= & S_{1} \cdot\left(S_{2} \times S_{3}\right)=\frac{1}{2 \mathrm{i}}\left[S_{1+}\left(S_{2 z} S_{3-}-S_{2-} S_{3 z}\right)+S_{1-}\left(S_{2+} S_{3 z}-S_{2 z} S_{3+}\right)\right. \\
& \left.+S_{1 z}\left(S_{2-} S_{3+}-S_{2+} S_{3-}\right)\right]
\end{aligned}
$$


which has the eigenvalue $\frac{1}{4} \sqrt{3}$ for the states $\left|\lambda,-\frac{1}{2}\right\rangle$ and $\left|\lambda^{-1},+\frac{1}{2}\right\rangle$ of (3) and the eigenvalue $-\frac{1}{4} \sqrt{3}$ for the other two. So it is easily verified that the operator $B_{z}$ defined by

$$
\begin{aligned}
B_{z} & =-\frac{8}{3} \sqrt{3}\left(S_{1 z}+S_{2 z}+S_{3 z}\right) A \\
& =\frac{\mathrm{i} \sqrt{3}}{3}\left(-S_{1+} S_{2-}+S_{1-} S_{2+}-S_{2+} S_{3-}+S_{2-} S_{3+}-S_{3+} S_{1-}+S_{3-} S_{1+}\right)
\end{aligned}
$$

has the eigenvalue +1 for the states $\left|\lambda, \pm \frac{1}{2}\right\rangle$ and the eigenvalue: -1 for $\left|\lambda^{-1}, \pm \frac{1}{2}\right\rangle$.

Consequently $B_{z}$ is immediately connected with the rotational character of the state. It is this operator in terms of which the rotational correlation (RC) will be defined. One considers two equilateral triangles like the one depicted in fig. 2; the correlation of the corresponding $B_{z}$ operators defines their $\mathrm{RC}$. The operator $B_{z}$ may be considered to be the $z$-component of a vector operator $B$. Instead of the correlation of the $B_{z}$ one may also consider $\left\langle\boldsymbol{B}_{\mathrm{I}} \cdot \boldsymbol{B}_{\mathrm{II}}\right\rangle$ for two triangles I and II. For a singlet state, however, the last correlation function simply equals $3\left\langle B_{1 z} B_{11 z}\right\rangle$, for reasons of symmetry.

For the states in (7) with values of $x(\lambda), y(\lambda), x\left(\lambda^{-1}\right)$ and $y\left(\lambda^{-1}\right)$ given by (8) one easily proves that they are eigenstates of the operators $B_{z}$ for both triangles 423 and 421 . The two eigenvalues equal 1 for the state $|\lambda\rangle$ and -1 for the state $\left|\lambda^{-1}\right\rangle$. In the following we only consider correlation functions of $B_{z}$ operators of disjunct triangles, because these operators do not commute for triangles that have one or two vertices in common.

\section{Ground states of systems of 6, 7 and 12 spins}

For 6 and 7 spins we consider configurations as shown in fig. 4. Again the sites are numbered and the sublattices are indicated by the lattice $a, b$ and $c$. The ground states for these two systems have rotational symmetry around the three

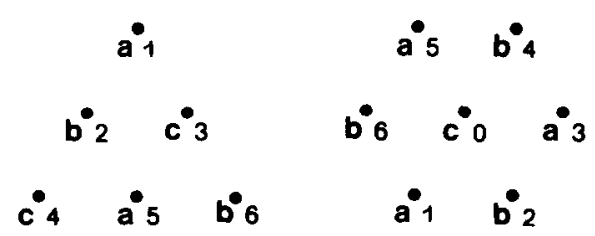

Fig. 4. Configurations of 6 and 7 spins. 
and sixfold axes respectively. In contradistinction to the example of 12 spins the two systems are supposed not to be obcy periodic boundary conditions; e.g. the spins 1,4 and 6 in the system of 6 spins only have two neighbours. The ground state of the 6-spin system turns out to be twofold and one of the corresponding states may be written as a linear combination of a $|\lambda\rangle$ and a $\left|\lambda^{-1}\right\rangle$ state. The ground state of the 7-spin system is twofold because of Kramers' degeneracy.

The clcmentary kets are written as in the examples with 3 and 4 spins; the position of a + or - sign in a ket corresponds with the numbers $1-6$ or, $0,1-6$ respectively. The kets may be grouped according to the rotational symmetry: each group is represented by one ket only.

\subsection{System of 6 spins}

In table III we have given the coefficients of the elementary kets in the non-normalized ground states $|\lambda\rangle$ and $\left|\lambda^{-1}\right\rangle$. In the last column we have given the number of states in the corresponding group, which is represented by one single ket. The state

$$
|\rangle=\frac{1}{6}\left[|\lambda\rangle-\left|\lambda^{-1}\right\rangle\right]
$$

is one of the ground states for the 6-spin system. The ground state energy equals -9 . The other ground state will not be given here because it is not interesting for our argument. The expcctation value of the energy for the state $|\lambda\rangle$, defined by table III, equals

$$
\frac{\langle\lambda|H| \lambda\rangle}{\langle\lambda \mid \lambda\rangle}=-\frac{51}{7}=-7.286
$$

An optimalized $\lambda$ state, according the definitions of section 2 gives a value -8.046 , which differs only $11 \%$ from the exact value. For a 4-spin system the corresponding difference cquals

$$
\frac{7-5.372}{7} \times 100 \%=23 \% \text {. }
$$

Table III

Ground state of the 6 spin system

\begin{tabular}{llll}
\hline \multicolumn{1}{l}{223456} & $|\lambda\rangle$ & $\left|\lambda^{-1}\right\rangle$ \\
\hline 1$\rangle=|-+++--\rangle$ & $\lambda^{-1}$ & $\lambda$ & 3 \\
$|2\rangle=|--+++-\rangle$ & $\lambda$ & $\lambda^{-1}$ & 3 \\
$|3\rangle=|+---++\rangle$ & $\lambda$ & $\lambda^{-1}$ & 3 \\
$|4\rangle=|++-+-+\rangle$ & $\lambda^{-1}$ & $\lambda$ & 3 \\
$|5\rangle=|-++-+-\rangle$ & 1 & 1 & 1 \\
$|6\rangle=|+--+-+\rangle$ & 1 & 1 & 1 \\
\hline
\end{tabular}




\subsection{Systems of 7 spins}

Straightforward calculation gives us the ground state of the 7-spin system. The total spin $S$ of this odd numbered system equals $\frac{1}{2}$, and the total spin of the ring of the spins 1-6 equals 1 . Coupling of this last spin with the central spin gives the ground state with total energy $-8-2 \sqrt{ } 5$.

The ground state has sixfold symmetry around the central axis of the hexagon and the kets may be ordered into groups of 6,2 or 3 members, as shown in table IV, in which the amplitudes are given. All groups are represented by one ket. The ground state given here corresponds with $S=\frac{1}{2}$ and $M=\frac{1}{2}$, one of a Kramers doublet.

If one constructs a $|\lambda\rangle\left(\left|\lambda^{-1}\right\rangle\right)$ state, which will not be done explicitly in this work, one finds complex phases that are in accordance with those of the groundstate $\mid>$ that again may be represented by a linear combination: $c\left[|\lambda\rangle+\left|\lambda^{-1}\right\rangle\right]$, with the single exception of the phase of the group $|1\rangle$. Taking real amplitudes in the linear combination one finds the phases indicated in the column: $|\lambda\rangle+\left|\lambda^{-1}\right\rangle$. One should bear in mind, however, that the group $|1\rangle$ corresponds with the smallest amplitude. For this example we have not determined the expectation value of the state $|\lambda\rangle\left(\left|\lambda^{-1}\right\rangle\right)$, because the coefficient of the states corresponding to $|1\rangle$ could not be properly determined.

\subsection{Systems of 12 spins}

For the system of 12 spins with periodic boundary conditions the ground state energy and the 2-spin correlation functions were already determined by Marland and Betts ${ }^{15}$ ). We also determined the correlation function for the operator $B_{z}$ of different triangles, in the ground state, which is a singlet state and corresponds with the identical representation of the translation group. For a proper definition of the relevant triangles we have to number the spins. This is done in fig. 5 in which also the sublattices are indicated. Because of periodic boundary conditions it is not relevant which configuration of the 12 spins is chosen, the one of fig. 5, or the diamond of Marland and Betts.

Table IV

Ground state of the 7-spin system.

\begin{tabular}{clcc}
\hline \multicolumn{1}{|c|}{123456} & \multicolumn{1}{c}{\rangle} & $|\lambda\rangle+\left|\lambda^{-1}\right\rangle$ \\
\hline$|1\rangle=|++++---\rangle$ & $\frac{1}{12}-\frac{1}{20} \sqrt{5}$ & + & 6 \\
$|2\rangle=|+++-+--\rangle$ & $\frac{1}{30} \sqrt{5}$ & + & 6 \\
$3\rangle=|+++--+-\rangle$ & $\frac{1}{30} \sqrt{5}$ & + & 6 \\
$|4\rangle=|++-+-+-\rangle$ & $-\frac{1}{4}-\frac{1}{20} \sqrt{5}$ & - & 2 \\
$|5\rangle=|-++++--\rangle$ & $-\frac{1}{6}+\frac{1}{30} \sqrt{5}$ & - & 6 \\
$|6\rangle=|-+++-+-\rangle$ & $\frac{1}{6}+\frac{3}{30} \sqrt{5}$ & + & 6 \\
$|7\rangle=|-++-++-\rangle$ & $-\frac{2}{15} \sqrt{5}$ & - & 3 \\
\hline
\end{tabular}




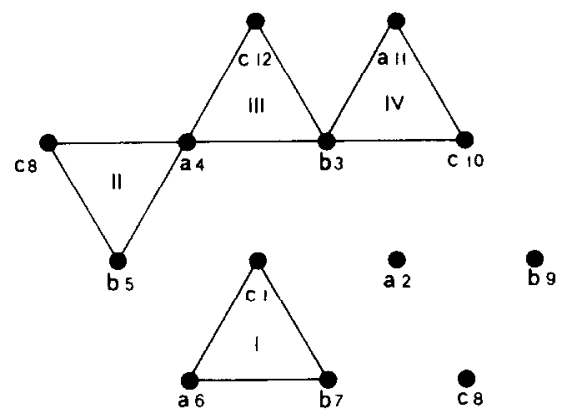

Fig. 5. Configuration of 12 spins.

There are different types of pairs of disjunct triangles, which may be represented by pairs of the following set:

$$
\text { 6.7.1 (I), } 4.5 .8(\mathrm{II}), \quad 4.3 .12 \text { (III) and } 11.3 .10(\mathrm{IV})
$$

The order of the indices $6,7,1$, e.g., is always chosen in such a way that the first index corresponds with a point of the $a$ sublattice, the second one with the $b$ lattice and the last one with the $c$ lattice. For the definition of the operators $B_{z}$, according to (13) the first spin always corresponds with the first index, etc. In this way we arrive at a unique definition of the operators $B_{\mathrm{I} z}$, etc., the correlations of which are given in table $\mathrm{V}$.

\begin{tabular}{lc}
\multicolumn{2}{c}{ Table V } \\
Correlation of $B_{z}$ operators. \\
\hline$\left\langle B_{\mathrm{Iz}} B_{\mathrm{IIz}}\right\rangle$ & 0.3492 \\
$\left\langle B_{\mathrm{Iz}} B_{\mathrm{III} z}\right\rangle$ & 0.3281 \\
$\left\langle B_{\mathrm{I} z} B_{1 \mathrm{~V} z}\right\rangle$ & 0.3319 \\
\hline
\end{tabular}

The coherence of the three correlations is rather remarkable and supports the suggestion of introducing $B_{z}$ as an order parameter. It should be taken into consideration that the maximum value of these correlations equals 1 , but this value could not be realized for a state that is translationally invariant, because one cannot construct a state in which all triangles are in one of the states (3), and this condition is necessary for the operators $B_{z}$ to have their maximum absolute value.

\section{Discussion of the results}

The results for the 4 and 12 spin system clearly show the phenomenon of RC. For the systems with a small number of spins, however, it is rather difficult to define in a simple way the rotational correlation function, because the corres- 
ponding operators do not commute. For all examples, with the exception of the 7 -spin system, we could show that (one of) the ground state(s) can be written as a linear combination: $c\left[|\lambda\rangle \pm\left|\lambda^{-1}\right\rangle\right]$. In the $|\lambda\rangle$ state, for instance, all triangles are in a state of the type:

$$
c_{0}|+--\rangle+c_{1} \lambda|-+-\rangle+c_{2} \lambda^{-1}|--+\rangle, \quad c_{0}, c_{1}, c_{2} \text { real } \geqslant 0,
$$

or

$$
c_{0}^{\prime}|-++\rangle+c_{1}^{\prime} \lambda|++-\rangle+c_{2}^{\prime} \lambda^{-1}|+-+\rangle, \quad c_{0}^{\prime}, c_{1}^{\prime}, c_{2}^{\prime} \text { real } \geqslant 0,
$$

or in a quartet state $|+++\rangle$ or $|---\rangle$. It is impossible that all triangles are in a $\lambda$ state, and we have the opinion that an asymptotic ground state for large systems may be constructed, that is a linear combination of states in which a maximum number of triangles is in a $\lambda$ state, corresponding with the lowest possible energy for that triangle. It stands to reason that $|\lambda\rangle$ and $\left|\lambda^{-1}\right\rangle$ are asymptotically orthogonal eigenstates of $H$, thus showing RSB.

In concluding our work, our attention was drawn to work by Miyashita ${ }^{17}$ ). The point of view of this author, however, is quite different from ours because he considers an ordering for which there exists a net sublattice magnetization. He succeeds in calculating an upper bound for the ground state energy of systems of arbitrary sizc.

States of the type $|\lambda\rangle$ or $\left|\lambda^{-1}\right\rangle$ show characteristic complex phase relations between the coefficients of their components: this may be considered as a generalization of an exact property proved by Marshall ${ }^{18}$ ) for the "standard" antiferromagnetic systems, for which $t w o$ sublattices may be distinguished. Here the interchange of a + and $a-$ spin on neighbouring sites always results in a change of sign of the corresponding elementary kets, which are eigenstates of all the $z$-components of the individual spins. Essential for this property is the fact that all polygons of nearest neighbour pairs have an even number of vertices, whereas in the triangular lattice the smallest polygon is a triangle. This property of the triangular lattice suggests the phases rcalized in the $|\lambda\rangle$ states, which, however, could only be constructed as asymptotic states for large systems.

\section{Acknowledgement}

The authors wish to thank Dr. W. Magnus for critical reading of the manuscript.

\section{References}

1) E. Lieb, Th. Schultz and D. Mattis, Ann. Phys. 16 (1961) 407.

2) B.M. McCoy, Phys. Rev. 173 (1968) 531. 
3) T. Tonegawa, Solid State Commun. 40 (1981) 983.

4) J. Bonner and M.E. Fisher, Phys. Rev. 135 (1964) A640.

5) N.D. Mermin and H. Wagner, Phys. Rev. Lett. 17 (1966) 1133.

6) C.K. Majumdar and D.K. Ghosh, J. Math. Phys. 10 (1969) 1388, 1399.

7) C.K. Majumdar, J. Phys. C3 (1970) 911

8) P.M. van den Broek, Phys. Lett. 77A (1980) 261.

9) W.J. Caspers and W. Magnus, Physica 119A (1983) 291.

10) J. Goldstone, Nuovo Cimento 19 (1961) 154.

11) B.S. Shastry and B. Sutherland, Phys. Rev. Lett. 47 (1981) 964.

12) W.J. Caspers, K.M. Emmet and W. Magnus, J. Phys. A17 (1984) 2687.

13) G.H. Wannier, Phys. Rev. 79 (1950) 357.

14) K.A. Penson, R. Jullien and P. Pfeuty, J. Phys. C12 (1979) 3967.

15) L.G. Marland and D.D. Betts, Phys. Rev. Lett. 43 (1979) 1618.

16) A.R. Edmonds, Angular Momentum in Quantum Mechanics (Princeton Univ. Press, Prince ton, 1957).

17) S. Miyashita, J. Phys. Soc. Japan 53 (1984) 44.

18) W. Marshall, Proc. Roy. Soc. (London) A232 (1955) 48. 